\title{
Anabases
}

$\triangle N A B A S E S$ Traditions et réceptions de l'Antiquité

6 | 2007

Varia

\section{GAGGADIS-ROBIN, Les sarcophages païens du Musée de l'Arles antique}

Robert Sablayrolles

\section{OpenEdition}

Journals

Édition électronique

URL : http://journals.openedition.org/anabases/3433

DOI : $10.4000 /$ anabases.3433

ISSN : 2256-9421

\section{Éditeur}

E.R.A.S.M.E.

\section{Édition imprimée}

Date de publication : 1 octobre 2007

Pagination : 280-283

ISSN : 1774-4296

\section{Référence électronique}

Robert Sablayrolles, «V. Gaggadis-RoBin, Les sarcophages païens du Musée de l'Arles antique », Anabases

[En ligne], 6 | 2007, mis en ligne le 01 janvier 2012, consulté le 21 septembre 2020. URL : http://

journals.openedition.org/anabases/3433 ; DOI : https://doi.org/10.4000/anabases.3433

Ce document a été généré automatiquement le 21 septembre 2020

(c) Anabases 


\title{
V. GAGGADIS-ROBIN, Les sarcophages païens du Musée de l'Arles antique
}

\author{
Robert Sablayrolles
}

\section{RÉFÉRENCE}

V. GAGGADIS-ROBIN, Les sarcophages païens du Musée de l'Arles antique, Arles, éd. du musée de l'Arles et de la Provence antiques, 2005, $542 \mathrm{p}$.

40 euros, ISBN : 2-9516385-6-6

1 Le musée de l'Arles antique poursuit l'œuvre de publication de ses collections et, après le volume consacré aux lampes, offre un catalogue des sarcophages païens, dont la réalisation a été confiée à Madame Vassiliki Gaggadis-Robin, chargée de recherche au CNRS dans l'équipe du Centre Camille Jullian. Les éditions du musée de l'Arles et de la Provence antiques ont su se donner les moyens d'une publication luxueuse, à la hauteur de la collection des sarcophages païens, la plus importante en France après celle du musée du Louvre, et de la remarquable présentation qui en est faite dans ce musée qui doit tant à son fondateur, J.-M. Rouquette.

2 Le catalogue adopte le plan classique d'une histoire de la collection, d'une analyse individuelle des quatre-vingt-douze pièces recensées et d'une synthèse sur les principaux acquis de cette étude détaillée. Deux annexes sur les techniques d'analyse des marbres utilisées pour déterminer l'origine des pièces (mesures isotopiques et cathodoluminescence), une abondante bibliographie et une série d'indices, bien construits et très utiles, complètent l'ouvrage. Chaque notice est abondamment illustrée de photographies en couleur d'excellente qualité, même pour les pièces fragmentaires, et les sarcophages les plus riches, notamment celui de la légende de Phèdre et Hippolyte, joyau de la collection, bénéficient d'une profusion bien venue de photographies de détail. La présentation des notices est elle aussi particulièrement soignée, avec de discrets onglets colorés qui marquent le passage d'une rubrique à une 
autre et un jeu de tailles différentes pour les caractères mettant en valeur les parties essentielles des descriptions.

3 L'ordre de présentation des sarcophages induit implicitement une des conclusions essentielles de l'ouvrage et révèle le principal centre d'intérêt de l'auteur : les pièces sont présentées par ateliers d'origine (l'Asie Mineure, l'Attique, Rome et l'Italie, les ateliers locaux) et, à l'intérieur de ces catégories, par sujet du décor (sarcophages à guirlandes, à arcades, "thèmes inspirés de la vie humaine", titre dans lequel vie humaine aurait pu être avantageusement remplacé par « vie quotidienne » ou « vie du défunt ", scènes mythologiques, sujets décoratifs, «thèmes saisonniers »- qui sont en fait des représentations des saisons -, etc.). Le grand mérite de Madame V. GaggadisRobin et des scientifiques qui l'ont assistée dans les analyses des marbres est, en effet, d'avoir mis en lumière la grande part des ateliers micrasiatiques dans l'approvisionnement d'Arles, en rendant à ceux-ci nombre de productions considérées jusqu'alors comme romaines ou italiennes. La primauté chronologique de ces ateliers, affirmée par l'auteur dans ses conclusions, est moins assurée : elle ne repose en fait que sur la datation des deux premières pièces du catalogue, attribuées à la première moitié du $\mathrm{II}^{\mathrm{e}}$ siècle et qui se trouvent être, en conséquence, les plus anciennes de la collection. Il suffirait d'un seul sarcophage italien plus ancien pour remettre l'hypothèse en question. Le lecteur est également surpris qu'un couvercle et une cuve ( $\mathrm{n}^{\circ} 4$ et 71$)$, tous deux en marbre de Proconnèse et tous deux trouvés le même jour au même endroit dans les alluvions du Rhône, soient séparés dans le catalogue et considérés comme issus d'ateliers différents, le couvercle d'Asie Mineure, la cuve d'Arles, alors même que l'auteur semble considérer que, malgré une légère différence de taille, ils appartenaient à un même monument.

4 L'étude de Madame V. Gaggadis-Robin est essentiellement une analyse d'historienne de l'art, ce qui est bien naturel pour une collection de cette ampleur et de cette valeur. Les descriptions des techniques d'exécution, l'observation des traces d'outils sont détaillées et précises, les motifs et les scènes des décors sont replacés dans des séries qui témoignent, comme la bibliographie, de la parfaite érudition de Madame V. GaggadisRobin dans la spécialité. Certaines attributions paraissent audacieuses, tant le fragment qui subsiste conserve peu de données pour restituer l'ensemble ( $n^{\circ} 10,35$ ou 39, entre autres), mais d'autres études sont particulièrement solides et fermement étayées sur un corpus de références très étoffé, une des plus remarquables en la matière étant l'interprétation du décor original d'un couvercle, attribué de façon convaincante à un défunt propriétaire de chevaux de courses ( $\left.n^{\circ} 54\right)$. La chronologie proposée pour ces sarcophages est déterminée, de façon presque exclusive, sur des critères techniques (traces des outils) ou esthétiques (nature et agencement des scènes, éléments de décor). Sans doute parlera-t-elle plus aux spécialistes qu'aux profanes, qu'elle laissera parfois sceptiques. Le sarcophage de la légende de Phèdre et Hippolyte, daté des années 230-240 par un des prédécesseurs de l'auteur, est placé par elle «autour de 250 ». En dépit de l'exceptionnelle richesse du décor, brillamment commenté et illustré, et malgré une subtile et convaincante démonstration de Madame Gaggadis-Robin, qui prouve que cette pièce issue d'un atelier attique a été retouchée par un artisan d'Arles pour refaire le personnage du commanditaire, il est difficile de croire que des critères fondés sur la seule analyse esthétique autorisent une telle précision.

5 L'épigraphie, présente sur quatorze cuves et deux couvercles, n'est que rarement mise à contribution pour la chronologie et presque toujours, dans ces cas-là, sans référence 
explicite aux critères utilisés (onomastique, présence ou absence de l'invocation aux dieux Mânes, etc.). On regrette également, dans ce domaine, l'absence d'une restitution latine du texte avec application des signes diacritiques traditionnels (parenthèses notamment pour le développement des abréviations). Madame V. Gaggadis-Robin dit bien dans sa conclusion que les monuments épigraphes, en trop petit nombre dans son corpus, "ne sont pas d'une grande utilité » et qu'il faut attendre la publication des inscriptions d'Arles par M. Heijmans dans la série des Inscriptions Latines de Narbonnaise. La consultation du seul CIL, sans doute vieilli et qui sera heureusement complété, sinon remplacé, par les publications à venir, aurait cependant permis de préciser que l'inscription métrique de Iulia Lucina $\left(\mathrm{n}^{\circ} 74\right)$ était composée d'hexamètres dactyliques, ou que la lecture de l'inscription de Sex. Alfius Vitalis $\left(n^{\circ} 68\right)$ n'était pas aussi claire et assurée que le laisse penser la présentation du catalogue. Enfin, les mêmes vieux auteurs du CIL (XII, 735) font leur, sans doute avec raison, l'hypothèse, encore plus ancienne, de l'érudit avignonnais E. Calvet, qui voyait dans le mot birbilitan de l'inscription $\mathrm{n}^{\circ} 80 \mathrm{du}$ catalogue, non pas un nom propre, mais bien un adjectif Birbiltan(a). Cet adjectif pourrait évoquer l'origine de l'un des personnages : la colonie de Bilbilis, en Tarraconaise. L'inscription, dans la traduction qui en est donnée, suppose, en outre, un solécisme, possible, du lapicide ou du commanditaire, mais qu'il faudrait alors signaler ; une autre traduction du texte permettrait de ne pas recourir à cette solution du solécisme.

6 Les descriptions techniques et esthétiques des monuments dans les notices sont, comme il a déjà été rappelé, précises, appropriées et détaillées. L'écriture des chapitres de synthèse, introduction ou conclusion, souffre en revanche d'un français familier parfois mal venu dans une publication de cette qualité. Au néologisme « massivité » des monuments, on aurait préféré caractère massif ou lourdeur, "l'appellation fréquente de l'œuvre " signifie, semble-t-il, sa « désignation traditionnelle », et la multiplication des adjectifs et des superlatifs dans les épitaphes latines, effectivement indice d'une chronologie tardive, ne mérite pas, même entre guillemets, le qualificatif de style "enflé »: il vaudrait mieux dire style ampoulé, pompeux ou emphatique. C'est aussi, sans doute, à une relecture insuffisante qu'il faut attribuer l'implantation de la Mésopotamie, sur la carte de l'Empire romain donnée au début de l'ouvrage, aux abords de la mer Rouge, sur les côtes de l'Arabie.

7 Ces approximations, dommageables, ne doivent pas faire oublier l'essentiel : la série des sarcophages païens du musée de l'Arles antique est désormais dotée d'une publication digne du niveau de la collection et des espaces dans lesquels elle est exposée. L'analyse d'histoire de l'art y est remarquablement conduite, solidement étayée sur un corpus de références exhaustif et les conclusions qui en sont tirées en matière d'esthétique et de technique, mais aussi de circuits économiques des œuvres d'art, sont novatrices et convaincantes. 


\section{AUTEURS}

\section{ROBERT SABLAYROLLES}

Université de Toulouse II-Le Mirail

sablayro@univ-tlse2.fr 